\title{
MS5802
}

\section{Mechanics of rolling of nanoribbon on tube and sphere}

\section{施兴华 ${ }^{1}$ ， 殷其放 ${ }^{1}$}

\section{1. 中国科学院力学研究所, 北京 100190}

E-mail: shixh@imech.ac.cn

The configuration of graphene nano-ribbon (GNR) assembly on carbon nanotube (CNT) and sphere is studied through theoretical modeling and molecular simulation. The GNR can spontaneously wind onto the CNT due to van der Waals (vdW) interaction and form two basic configurations: helix and scroll. The final configuration arises from the competition among three energy terms: the bending energy of the GNR, the vdW interaction between GNR and CNT, the vdW between the GNR itself. We derive analytical solutions by accounting the three energy parts, with which draw phase diagrams and predict the final configuration (helix or scroll) based on the selected parameters. The molecular simulations are conducted to verify the model with the results agree well with the model predicted. Our work can be used to actively control and transfer the tube-like nanoparticles and viruses as well as to assemble ribbon-like nanomaterials.

Keywords: graphene nanoribbon;nanoscroll;

\section{Preferred Presentation Type:}

